\title{
The vascular bed in COPD: pulmonary hypertension and pulmonary vascular alterations
}

\author{
Seiichiro Sakao', Norbert F. Voelkel ${ }^{2}$ and Koichiro Tatsumi ${ }^{1}$
}

Affiliations: 'Dept of Respirology (B2), Graduate School of Medicine, Chiba University, Chiba, Japan. ${ }^{2}$ Victoria Johnson Center for Obstructive Lung Diseases and Pulmonary and Critical Care Medicine Division, Virginia Commonwealth University, Richmond, VA, USA.

Correspondence: Seiichiro Sakao, Dept of Respirology (B2), Graduate School of Medicine, Chiba University, 1-8-1 Inohana, Chuo-ku, Chiba 260-8670, Japan. E-mail: sakaosafaculty.chiba-u.jp

ABSTRACT The loss of pulmonary vessels has been shown to be related to the severity of pulmonary hypertension in patients with chronic obstructive pulmonary disease (COPD). The severity of hypoxaemia is also related to pulmonary hypertension and pulmonary vascular resistance in these patients, suggesting that the hypoxic condition probably plays an important role in this form of pulmonary hypertension.

However, pulmonary hypertension also develops in patients with mild COPD without hypoxaemia. Oxygen supplementation therapy often fails to reverse the pulmonary hypertension in these COPD patients, thus suggesting that the pulmonary vascular alterations in those patients may involve different sites of the pulmonary vasculature or a different form of vascular remodelling. It has recently been demonstrated that pulmonary vascular remodelling, resulting in pulmonary hypertension in COPD patients, can develop independently from parenchymal destruction and loss of lung vessels.

We wonder whether the changes in the lung microenvironment due to hypoxia and vessel loss have a causative role in the development of pulmonary hypertension in patients with COPD. Herein we review the pathobiological features of the pulmonary vasculature in COPD patients and suggest that pulmonary hypertension can occur with and without emphysematous lung tissue destruction and with and without loss of lung vessels.

\section{$@$ ERSpublications}

$\mathrm{PH}$ in COPD can occur with and without emphysematous lung tissue destruction and with and without lung vessel loss http://ow.ly/uOD5v

\section{Introduction}

Chronic obstructive pulmonary disease (COPD) is generally acknowledged to be a lung disease that presents with extrapulmonary manifestations, including skeletal muscle dysfunction, weight loss and osteoporosis [1]. COPD is characterised by chronic airflow limitation owing to lung parenchymal destruction and a narrowed airway calibre, frequently associated with alveolar hypoxia [2]. It is generally recognised that a loss of pulmonary vessels may contribute to secondary pulmonary hypertension in COPD, although its precise pathogenesis has not been fully elucidated. In truth, diminution and narrowing of the pulmonary vessels by angiography has been shown in patients with COPD [3, 4], and a diminished number of small pulmonary vessels, assessed by chest computed tomography (CT) scanning, contributes to the severity of pulmonary hypertension in patients with COPD [5].

Received: Oct 292013 | Accepted after revision: Nov 182013

Support statement: This study was supported by research grants from the Respiratory Failure Research Group (24-046) and the Cardiovascular Diseases Research Group (24-57) from the Ministry of Health, Labour and Welfare (Japan).

Conflict of interest: None declared.

Provenance: Submitted article, peer reviewed.

Copyright OERS 2014. ERR articles are open access and distributed under the terms of the Creative Commons Attribution Non-Commercial Licence 4.0. 
In addition, the severity of hypoxaemia has been related to the increase in pulmonary arterial pressure (PAP) and pulmonary vascular resistance in patients with COPD [6, 7]. The 4th World Symposium on Pulmonary Hypertension classified COPD-related pulmonary hypertension under group 3 "pulmonary hypertension associated with lung disease and/or hypoxia", thus indicating that hypoxic conditions play an important role in this form of pulmonary hypertension [8]. Alveolar hypoxia induces regional pulmonary vasoconstriction, which plays an important role in the maintenance of the ventilation/perfusion balance $[9,10]$. For instance, in healthy high-altitude natives, long-term alveolar hypoxia can cause pulmonary vascular alterations, including medial wall thickening and muscularisation of nonmuscularised arterioles [11]. These are caused by the hypoxic pulmonary vasoconstriction (HPV) associated with high fluid shear stress (fig. 1). These vascular changes are potentially reversible, because when such subjects return to sea level for a sufficient amount of time, the shear stress decreases and pulmonary hypertension is ameliorated [12].

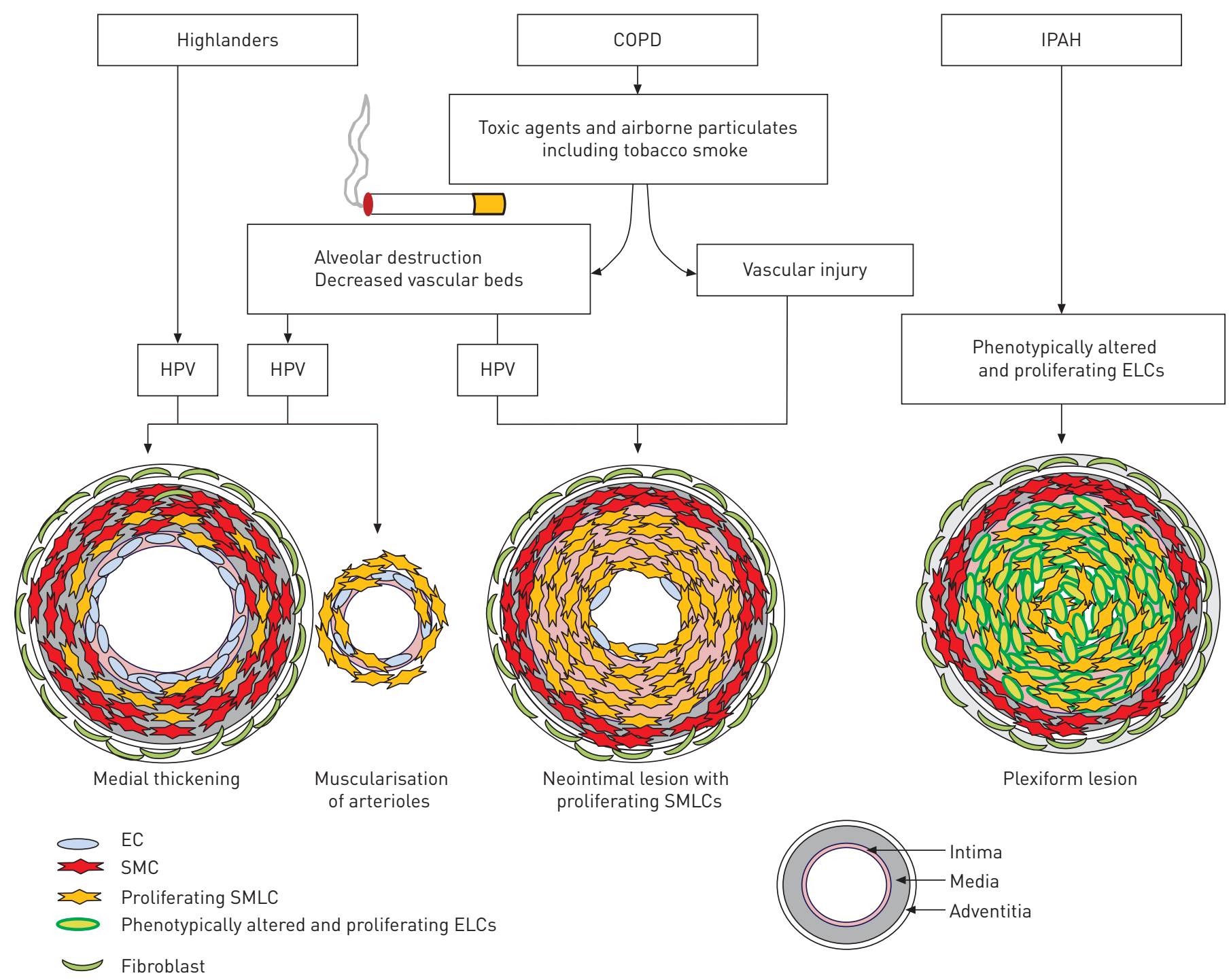

FIGURE 1 A hypothetical mechanism underlying the pulmonary vascular remodelling in highlanders, chronic obstructive pulmonary disease (COPD) patients and idiopathic pulmonary arterial hypertension (IPAH) patients. In healthy highlanders, long-term alveolar hypoxia can cause pulmonary vascular alterations, including medial wall thickening and muscularisation of nonmuscularised arterioles with proliferative smooth muscle-like cells (SMLCs). These are caused by the high fluid shear stress owing to hypoxic pulmonary vasoconstriction (HPV), resulting in an elevated pulmonary arterial pressure. In the patients with COPD, both vascular injuries caused by toxic agents and airborne particulates, including tobacco smoke, and the haemodynamic changes induced by the hypoxic condition resulting from the parenchymal destruction and the decreased vascular bed, may have a role in inducing the neointimal lesions consisting of proliferative SMLCs in the pulmonary muscular arteries. These lesions are different from the pulmonary vascular alterations seen in highlanders and in the pulmonary hypertension models induced by chronic hypoxic exposure alone. In the patients with IPAH, the defining pulmonary vascular alterations are complex vascular lesions consisting of phenotypically altered and proliferating endothelial-like cells (ELCs). The distinction between the pathological features in IPAH and COPD seems to largely be the presence or absence of complex vascular lesions with phenotypically altered and proliferating ELCs. EC: endothelial cell; SMC: smooth muscle cell. 
It has been reported that the pulmonary vascular remodelling in patients with advanced COPD resembles the lesions observed in healthy high-altitude natives $[13,14]$. However, pulmonary vascular remodelling also develops in patients with nonhypoxaemic mild COPD [15]. Moreover, oxygen therapy often fails to reverse the pulmonary hypertension in COPD patients [16], suggesting that the pulmonary vascular remodelling in those patients includes different components not explained by hypoxic vasoconstriction.

Experimentally, tobacco smoke can cause the development of pulmonary vascular alterations, suggesting that hypoxia may not be a sufficient explanation for pulmonary arterial remodelling in COPD [17, 18]. These reports suggest that the vascular lesions caused by tobacco smoke were probably different from the alterations caused by hypoxia. The pulmonary hypertension arising due to pulmonary vascular remodelling in COPD patients could develop independently from the emphysematous parenchymal destruction and lung vessel loss. SEIMETZ et al. [17] related the development of pulmonary hypertension and lung vascular remodelling to the inducible nitric oxide synthase (iNOS) pathway in bone marrow-derived cells, and the development of emphysema to the iNOS pathway in non-bone marrow-derived cells. FerRER et al. [18] demonstrated that cigarette smoke-related endothelial dysfunction might induce proliferation of smooth muscle-like cells (SMLCs), resulting in neointimal lesions in patients with COPD, and that the remodelling caused by cigarette smoke might be enhanced by hypoxic conditions.

Clinically, MinAI et al. [19] emphasised that most patients with COPD had mild-to-moderate pulmonary hypertension and normal cardiac function. Severe pulmonary hypertension (defined as mean PAP $>40 \mathrm{mmHg}$ ) in COPD patients is rare, and the severity of pulmonary hypertension seems to be disproportionate to the respiratory impairment [19]. Furthermore, in the COPD patients with mild-tomoderate hypoxaemia, PAP seems to progress slowly, at a rate of $0.4 \mathrm{mmHg}$ per year [20].

This review asks whether the lung microenvironment of hypoxia and vessel loss plays a causative role in the development of pulmonary hypertension in patients with COPD. We also wonder why the pulmonary hypertension in patients with COPD tends to be relatively mild and progresses slowly. As well as what the differences are in the development of vascular remodelling between pulmonary arterial hypertension (PAH), as classified under World Health Organization group 1, and pulmonary hypertension associated with COPD.

Herein we review the pathobiological features of the pulmonary vasculature in patients with COPD.

\section{Hypoxic conditions and reverse remodelling in COPD}

The destruction of the lung parenchyma and increased airway resistance induced by bronchial luminal obstruction with mucoid secretions can explain hypoxia in patients with COPD [2]. In hypoxic rodent models of pulmonary hypertension, the exposure to hypoxic conditions causes media thickening and muscularisation of nonmuscularised arteries with hyperproliferative smooth muscle cells (SMCs), resulting in pulmonary hypertension [21-24]. Histologically, features that closely resemble these pulmonary arterial alterations have been confirmed in the pulmonary vasculature of COPD patients, including lumen narrowing caused by increased medial thickness and muscularisation in arterioles [13, 14, 25]. This suggests that COPD patients may share one or several pathophysiological processes underlying the development of pulmonary vascular remodelling with chronic hypoxia rodent models [21-23]. Moreover, in COPD patients, pulmonary hypertension is usually slowly progressive and pulmonary hypertension is mild or moderate [19], as in the rat model [21, 24].

As described previously [23], the vascular lesions consisting of proliferating SMCs may potentially be reversible as in hypoxic rodent models of pulmonary hypertension, while in severe angio-obliterative forms of PAH, lesions with phenotypically altered endothelial-like cells (ELCs) are irreversible. Due to the reasons described above, the vascular lesions in patients with COPD, which share pathophysiological processes with chronic hypoxia rodent models, may potentially be reversible.

Further confirming the differences between pulmonary vascular lesions, the complex vascular lesions in a rat model of severe PAH, which is based on the subcutaneous injection of vascular endothelial growth factor (VEGF) receptor blockade (Sugen5416; SUGEN Inc., San Francisco, CA, USA) and the second hit of chronic hypoxia [26,27], have been shown to be irreversible [28]. In fact, the complex vascular lesions in patients with idiopathic PAH (IPAH) contain apoptosis-resistant [29-31], phenotypically altered and proliferating [26, 32, 33] and monoclonal endothelial cells [34], resulting in arterial occlusion that may be irreversible $[23,35]$. In IPAH patients, there are some complex vascular lesions, i.e. plexiform lesions, in the pulmonary vasculature, which are the hallmark of severe PAH. In contrast, most COPD patients do not have these lesions [6]. This may suggest that clinical features such as the mild-to-moderate pulmonary hypertension and slow pulmonary hypertension progression in COPD patients are attributable to the absence of these lesions and the absence of dysregulated ELCs (fig. 1). 
Intimal lesions of the pulmonary vasculature in COPD

Pulmonary vascular remodelling in patients with COPD is also characterised by an increased intimal thickness due to proliferating SMLCs in pulmonary muscular arteries $[25,36]$. This suggests that the intimal lesions with SMLCs have different pathological features when compared to the pulmonary vascular alterations seen in the chronic hypoxia pulmonary hypertension models. SMLCs are positive for anti- $\alpha$ smooth muscle actin (the SMC-specific marker) and vimentin (the mesenchymal-specific marker), and negative for desmin (the SMC-specific marker) [37]. The expression pattern of these markers suggests that they are a subpopulation of immature SMCs that may be critical for effective vascular remodelling [37, 38]. In addition, there is deposition of the extracellular matrix (ECM) in neointimal lesions in COPD patients [37], which is thought to lead to intimal hypertrophy [39]. However, the neointimal lesions in COPD patients do not demonstrate any phenotypically altered and proliferating ELCs, which is a different pathological feature when compared with the neointimal lesions seen in patients with IPAH (fig. 1). In fact, they occur in lesions in rats after the combination of pneumonectomy, with monocrotaline injection [40-42]. In this particular rodent model, the addition of increased blood flow, i.e. high shear stress caused by pneumonectomy apparently induces neointimal lesions in the pulmonary arteries [41]. However, pneumonectomised human patients show only medial thickening of the pulmonary arteries, but no neointimal lesions several years after the surgery [43]. Taken together, these facts suggest that increased shear stress in the pulmonary arteries, in combination with vascular injury, can induce neointimal lesions. In truth, tobacco smoke products may directly cause endothelial cell dysfunction, resulting in impaired release of endothelial nitric oxide synthase [18], increased expression of VEGF [44], and increased numbers of inflammatory cells [45]. Moreover, endothelial cell dysfunction-related factors have been shown, in principle, to be able to affect vascular SMC growth [46].

As mentioned earlier, the development of pulmonary vascular alterations in patients with COPD appears to be tobacco smoke-induced, independent of hypoxaemia and perhaps independent of lung parenchymal destruction [17, 18]. This suggests that there may be two types of vascular remodelling in patients with COPD, i.e. tobacco smoke-related remodelling and hypoxic vasoconstriction-related remodelling. In fact, pulmonary vascular remodelling also develops in patients with mild COPD who are not typically hypoxaemic [15]. However, regional hypoxia in nonventilated areas of the lung may be present in COPD patients [6], suggesting that regional vascular remodelling related to HPV may still exist, even in the absence of systemic hypoxaemia [47]. In patients with COPD, not only the vascular injury caused by tobacco smoke, but also the haemodynamic changes induced by HPV, may combine to induce intimal lesions in the pulmonary arteries consisting of proliferating SMLCs. Supporting this idea, the pulmonary vascular alterations induced by tobacco smoke in a rodent model are only characterised by muscularisation of the arterioles, but not increased intimal thickness consisting of proliferative SMLCs in the pulmonary muscular arteries [17]. Thus multiple "hits", including both vascular injury and high shear stress, may be essential for the development of the intimal alterations in the pulmonary muscular arteries of patients with COPD (fig. 1).

However, so far, no complex vascular lesions consisting of phenotypically altered and proliferating ELCs have been described in the lungs of patients with COPD (fig. 1).

\section{Oxygen supplementation therapy and lung vessel loss in COPD}

As mentioned earlier, some of the components of the pulmonary vascular alterations in COPD patients include hypoxia-related remodelling, such as medial wall thickening. In fact, hypoxic conditions induce proliferation of not only medial SMCs [21-24], but also adventitial fibroblasts [48]. Fibroblast proliferation induces deposition of the ECM in pulmonary vasculature in COPD patients [37], which results in decreased pulmonary vascular compliance [48].

Although oxygen supplementation therapy does not normalise the remodelled vessels in COPD patients [16], this therapy has been shown to improve PAP in COPD patients, increasing their exercise capacity and decreasing the frequency of exacerbations [49]. However, this therapy also reduces the ventilatory drive and can result in hypercapnia [50]. Therefore, strictly controlled oxygen use with arterial oxygen saturation measured by pulse oximetry in the $90-92 \%$ range is important to avoid hypercapnia [51]. This restricted oxygen supplementation may be the reason why oxygen therapy often fails to reverse the increased pulmonary arterial pressure in COPD patients [16]; another reason may be that there is a nonhypoxic vasoconstriction participating in the pulmonary hypertension of COPD patients.

Studies have shown that people living at high altitude have pulmonary hypertension with reversible medial wall thickening and muscularisation of nonmuscularised arterioles [11, 12]. Indeed, some components of pulmonary vascular remodelling in patients with COPD consist of medial wall thickening resembling that in healthy highlanders $[13,14]$. Therefore, if oxygen therapy can increase the oxygen tension in the destroyed 
lung units without causing ventilation/perfusion mismatch, reduced ventilatory drive and hypercapnia (in patients with COPD), some components of the pulmonary vascular alterations, including medial wall thickening (i.e. hypoxia-related remodelling), might be reversed.

The pathophysiological factors that lead to an elevated PAP in patients with COPD are compression of alveolar vessels due to hyperinflation, hypoxia-induced pulmonary vasoconstriction and the obliteration of the pulmonary vasculature [52]. However, the fact that $>60 \%$ of the total pulmonary arteries need to be obliterated or lost for pulmonary hypertension to develop suggests that the destruction of the lung capillary bed is not a major factor in the development of pulmonary hypertension in patients with mild COPD [53]. Obliteration of the pulmonary vasculature may not be the principal cause of the development of pulmonary hypertension in the early stages of COPD, but may contribute during the progressive stage of this disease. Although the loss of pulmonary vessels assessed by chest CT scans has been shown to be closely related to the severity of pulmonary hypertension in COPD patients [5], the degree of lung vessel loss may be related to the severity of parenchymal destruction. The pulmonary hypertension may not only be related to lung vessel loss, but also to the pulmonary vasoconstrictive reactivity and/or the severity of vascular remodelling.

\section{Conclusions}

In patients with COPD, vascular injury caused by toxic agents and airborne particulates, including tobacco smoke, and haemodynamic changes induced by hypoxic conditions resulting from parenchymal destruction and lung vessel loss are factors that influence regional lung blood flow behaviour. The combination of vascular injury and high shear stress may result in the formation of neointimal lesions $[6,25,54]$, which are different from the pulmonary vascular alterations seen in highlanders and the pulmonary hypertension models induced by chronic hypoxia alone (fig. 1).

The distinction between the pathological features of IPAH and COPD is attributable to the presence or absence of complex vascular lesions which are composed of phenotypically altered and proliferating ELCs (fig. 1).

\section{References}

1 Agustí AG, Noguera A, Sauleda J, et al. Systemic effects of chronic obstructive pulmonary disease. Eur Respir J 2003; 21: $347-360$.

2 Cosio Piqueras MG, Cosio MG. Disease of the airways in chronic obstructive pulmonary disease. Eur Respir J 2001; 18: Suppl. 34, 41s-49s.

Scarrow GD. The pulmonary angiogram in chronic bronchitis and emphysema. Proc R Soc Med 1965; 58: 684-687.

Jacobson G, Turner AF, Balchum OJ, et al. Vascular changes in pulmonary emphysema. The radiologic evaluation by selective and peripheral pulmonary wedge angiography. Am J Roentgenol Radium Ther Nucl Med 1967; 100: 374-396.

5 Matsuoka S, Washko GR, Yamashiro T, et al. Pulmonary hypertension and computed tomography measurement of small pulmonary vessels in severe emphysema. Am J Respir Crit Care Med 2010; 181: 218-225.

6 Chaouat A, Naeije R, Weitzenblum E. Pulmonary hypertension in COPD. Eur Respir J 2008; 32: 1371-1385.

7 Chatila WM, Thomashow BM, Minai OA, et al. Comorbidities in chronic obstructive pulmonary disease. Proc Am Thorac Soc 2008; 5: 549-555.

8 Badesch DB, Champion HC, Sanchez MA, et al. Diagnosis and assessment of pulmonary arterial hypertension. J Am Coll Cardiol 2009; 54: Suppl. 1, S55-S66.

9 Wang J, Weigand L, Foxson J, et al. $\mathrm{Ca}^{2+}$ signaling in hypoxic pulmonary vasoconstriction: effects of myosin light chain and Rho kinase antagonists. Am J Physiol Lung Cell Mol Physiol 2007; 293: L674-L685.

10 Park SJ, Yoo HY, Earm YE, et al. Role of arachidonic acid-derived metabolites in the control of pulmonary arterial pressure and hypoxic pulmonary vasoconstriction in rats. Br J Anaesth 2011; 106: 31-37.

11 Heath D, Williams D, Rios-Dalenz J, et al. Small pulmonary arterial vessels of Aymara indians from the Bolivian Andes. Histopathology 1990; 16: 565-571.

12 Penaloza D, Arias-Stella J. The heart and pulmonary circulation at high altitudes: healthy highlanders and chronic mountain sickness. Circulation 2007; 115: 1132-1146.

13 Magee F, Wright JL, Wiggs BR, et al. Pulmonary vascular structure and function in chronic obstructive pulmonary disease. Thorax 1988; 43: 183-189.

14 Wright JL, Petty T, Thurlbeck WM. Analysis of the structure of the muscular pulmonary arteries in patients with pulmonary hypertension and COPD: National Institutes of Health nocturnal oxygen therapy trial. Lung 1992; 70: 109-124.

15 Wright JL, Lawson L, Paré PD, et al. The structure and function of the pulmonary vasculature in mild chronic obstructive pulmonary disease. Am Rev Respir Dis 1983; 128: 702-707.

16 Timms RM, Khaja FU, Williams GW. Hemodynamic response to oxygen therapy in chronic obstructive pulmonary disease. Ann Intern Med 1985; 102: 29-36.

17 Seimetz M, Parajuli N, Pichl A, et al. Inducible NOS inhibition reverses tobacco-smoke-induced emphysema and pulmonary hypertension in mice. Cell 2011; 147: 293-305.

18 Ferrer E, Peinado VI, Castañeda J, et al. Effects of cigarette smoke and hypoxia on pulmonary circulation in the guinea pig. Eur Respir J 2011; 38: 617-627.

19 Minai OA, Chaouat A, Adnot S. Pulmonary hypertension in COPD: epidemiology, significance, and management: pulmonary vascular disease: the global perspective. Chest 2010; 137: Suppl. 6, 39S-51S.

20 Kessler R, Faller M, Weitzenblum E, et al. "Natural history" of pulmonary hypertension in a series of 131 patients with chronic obstructive lung disease. Am J Respir Crit Care Med 2001; 164: 219-224. 
21 Meyrick B, Reid L. Hypoxia-induced structural changes in the media and adventitia of the rat hilar pulmonary artery and their regression. Am J Pathol 1980; 100: 151-178.

22 Jones PL, Cowan KN, Rabinovitch M. Tenascin-C, proliferation and subendothelial fibronectin in progressive pulmonary vascular disease. Am J Pathol 1997; 150: 1349-1360.

23 Sakao S, Tatsumi K, Voelkel NF. Reversible or irreversible remodeling in pulmonary arterial hypertension. Am J Respir Cell Mol Biol 2010; 43: 629-634.

24 Jeffery TK, Wanstall JC. Pulmonary vascular remodeling: a target for therapeutic intervention in pulmonary hypertension. Pharmacol Ther 2001; 92: 1-20.

25 Barberà JA, Peinado VI, Santos S. Pulmonary hypertension in chronic obstructive pulmonary disease. Eur Respir J 2003; 21: 892-905.

26 Taraseviciene-Stewart L, Kasahara Y, Alger L, et al. Inhibition of the VEGF receptor 2 combined with chronic hypoxia causes cell death-dependent pulmonary endothelial cell proliferation and severe pulmonary hypertension. FASEB J 2001; 15: 427-438.

27 Abe K, Toba M, Alzoubi A, et al. Formation of plexiform lesions in experimental severe pulmonary arterial hypertension. Circulation 2010; 121: 2747-2754.

28 Bogaard HJ, Natarajan R, Mizuno S, et al. Adrenergic receptor blockade reverses right heart remodeling and dysfunction in pulmonary hypertensive rats. Am J Respir Crit Care Med 2010; 182: 652-660.

29 Wohrley JD, Frid MG, Moiseeva EP, et al. Hypoxia selectively induces proliferation in a specific subpopulation of smooth muscle cells in the bovine neonatal pulmonary arterial media. J Clin Invest 1995; 96: 273-281.

30 Yuan JX, Rubin LJ. Pathogenesis of pulmonary arterial hypertension: the need for multiple hits. Circulation 2005; 111: 534-538.

31 Lévy M, Maurey C, Celermajer DS, et al. Impaired apoptosis of pulmonary endothelial cells is associated with intimal proliferation and irreversibility of pulmonary hypertension in congenital heart disease. J Am Coll Cardiol 2007; 49: 803-810.

32 Sakao S, Taraseviciene-Stewart L, Lee JD, et al. Initial apoptosis is followed by increased proliferation of apoptosisresistant endothelial cells. FASEB J 2005; 19: 1178-1180.

33 Sakao S, Taraseviciene-Stewart L, Cool CD, et al. VEGF-R blockade causes endothelial cell apoptosis, expansion of surviving $\mathrm{CD}^{34+}$ precursor cells and transdifferentiation to smooth muscle-like and neuronal-like cells. FASEB J 2007; 21: 3640-3652.

34 Lee SD, Shroyer KR, Markham NE, et al. Monoclonal endothelial cell proliferation is present in primary but not secondary pulmonary hypertension. J Clin Invest 1998; 101: 927-934.

35 Sakao S, Tatsumi K, Voelkel NF. Endothelial cells and pulmonary arterial hypertension: apoptosis, proliferation, interaction and transdifferentiation. Respir Res 2009; 10: 95.

Naeije R, Barberà JA. Pulmonary hypertension associated with COPD. Crit Care 2001; 5: 286-289.

37 Santos S, Peinado VI, Ramírez J, et al. Characterization of pulmonary vascular remodelling in smokers and patients with mild COPD. Eur Respir J 2002; 19: 632-638.

38 Owens GK, Kumar MS, Wamhoff BR. Molecular regulation of vascular smooth muscle cell differentiation in development and disease. Physiol Rev 2004; 84: 767-801.

39 Jones PL, Cowan KN, Rabinovitch M. Tenascin-C, proliferation and subendothelial fibronectin in progressive pulmonary vascular disease. Am J Pathol 1997; 150: 1349-1360.

40 Stenmark KR, Meyrick B, Galie N, et al. Animal models of pulmonary arterial hypertension: the hope for etiological discovery and pharmacological cure. Am J Physiol Lung Cell Mol Physiol 2009; 297: L1013-L1032.

41 Okada K, Tanaka Y, Bernstein M, et al. Pulmonary hemodynamics modify the rat pulmonary artery response to injury. A neointimal model of pulmonary hypertension. Am J Pathol 1997; 151: 1019-1025.

42 Nishimura T, Faul JL, Berry GJ, et al. Simvastatin attenuates smooth muscle neointimal proliferation and pulmonary hypertension in rats. Am J Respir Crit Care Med 2002; 166: 1403-1408.

43 Rudolph AM, Neuhauser EB, Golinko RJ, et al. Effects of pneumonectomy on pulmonary circulation in adult and young animals. Circ Res 1961; 9: 856-861.

44 Santos S, Peinado VI, Ramirez J, et al. Enhanced expression of vascular endothelial growth factor in pulmonary arteries of smokers and patients with moderate chronic obstructive pulmonary disease. Am J Respir Crit Care Med 2003; 167: 1250-1256.

45 Domínguez-Fandos D, Peinado VI, Puig-Pey R, et al. Pulmonary inflammatory reaction and structural changes induced by cigarette smoke exposure in the guinea pig. COPD 2012; 9: 473-484.

46 Sakao S, Taraseviciene-Stewart L, Wood K, et al. Apoptosis of pulmonary microvascular endothelial cells stimulates vascular smooth muscle cell growth. Am J Physiol Lung Cell Mol Physiol 2006; 291: L362-L368.

47 Nicolls MR, Mizuno S, Taraseviciene-Stewart L, et al. New models of pulmonary hypertension based on VEGF receptor blockade-induced endothelial cell apoptosis. Pulm Circ 2012; 2: 434-442.

48 Tozzi CA, Christiansen DL, Poiani GJ, et al. Excess collagen in hypertensive pulmonary arteries decreases vascular distensibility. Am J Respir Crit Care Med 1994; 149: 1317-1326.

49 Kim V, Benditt JO, Wise RA, et al. Oxygen therapy in chronic obstructive pulmonary disease. Proc Am Thorac Soc 2008; 5: 513-518.

50 Kent BD, Mitchell PD, McNicholas WT. Hypoxemia in patients with COPD: cause, effects, and disease progression. Int J Chron Obstruct Pulmon Dis 2011; 6: 199-208.

51 Moloney ED, Kiely JL, McNicholas WT. Controlled oxygen therapy and carbon dioxide retention during exacerbations of chronic obstructive pulmonary disease. Lancet 2001; 357: 526-528.

52 Scharf SM, Iqbal M, Keller C, et al. Hemodynamic characterization of patients with severe emphysema. Am J Respir Crit Care Med 2002; 166: 314-322.

53 Wright JL, Churg A. Effect of long-term cigarette smoke exposure on pulmonary vascular structure and function in the guinea pig. Exp Lung Res 1991; 17: 997-1009.

54 Voelkel NF, Vandivier RW, Tuder RM. Vascular endothelial growth factor in the lung. Am J Physiol Lung Cell Mol Physiol 2006; 290: L209-L221. 